\title{
Three-Dimensional Analysis of Basic Formation Initialization Algorithms in Deep Space
}

\author{
M. Bikdash, F. Hadaegh, D. Scharf, and S. Ploen
}

\begin{abstract}
In this paper, we consider the problem of establishing Front-to-Front Sensor Lock (FFSL) between two spacecraft when the relative trajectory is not constrained to a plane that is normal to the sunline. We then provide a detailed three-dimensional analysis of the basic formation initialization algorithm, and introduce projection operations in which establishing FFSL in 3D is effectively reduced to analysis in a plane. Finally, we provide easily testable conditions whether in-plane searching will be suf ficient to guarantee FFSL and discuss a situation where in-plane search fails.
\end{abstract}

\section{INTRODUCTION}

Spacecraft formation flying has been recognized as a key enabling technology for NASA astrophysical and Earth science missions. NASA is deploying formation-based deepspace variable-baseline interferometers for high resolution imaging. The spacecraft in these formations must be able to communicate and measure accurately their relative states under various fault conditions. This requirement is accomplished using a special Autonomous Formation Flying (AFF) sensor described in [1]. When facing the AFF sensor on another spacecraft, the AFF sensor is capable of measuring accurately the relative position. When this occurs, we say that the two spacecraft have established Front-toFront Sensor Lock (FFSL). This may be needed under various scenarios. For instance, at initial deployment, or after a computer reset due to a solar flare.

The main difficulty in deep-space formation initialization is that a given spacecraft cannot determine its own inertial position with sufficient accuracy. Each spacecraft however can determine its own absolute attitude with great accuracy using onboard star trackers. An omnidirectional communication link between each spacecraft is assumed available.

Formation Initialization (FI), see [2,3] and references therein, is the process of first establishing communication between spacecraft and then obtaining accurate sensorbased estimates of relative translational states. FI may also include an active modification of these states (to avoid collisions, for instance), but this aspect is not considered in this paper. FI is complicated by the fact that the spacecraft

\footnotetext{
This work was partly conducted while M. Bikdash was visiting at JPL in June 2003 and June 2003. It is also partly supported by JPL Contract Number 1246600.

Dr. M. Bikdash is with the Department of Electrical and Computer Engineering, North Carolina A\&T State University, Greensboro, North Carolina, US. bikdash@ncat.edu

Drs. F. Hadaegh, D. Scharf, and S. Ploen are with the Jet Propulsion Loaboratory, California Institute of Technology, Pasadena, California, USA. \{Fred.Y.Hadaegh, dscharf, Scott.Ploen\}@jpl.nasa.gov
}

payload must remain behind its sun shield as to prevent thermal exposure. Hence, most attitude rotations must be about (or almost about) the sunline.

Scharf et al. [3] presented a complete FI algorithm for multiple spacecraft in deep space. For two spacecraft, which is the only case considered in this sequel, the algorithm is designed so that the boresights of the sensors remain anti-parallel at all times. Briefly, this algorithm consists of two stages. In the first stage, called In-Plane Search (IPS), the boresights are rotated (with the same angular speed) while remaining normal to the sunline; a process we refer to here as scanning. Since the beam pattern of the AFF sensor is conical (typically of halfangle $\gamma \approx 70^{\circ}$ ), there is a region of space, called the complementary cones (CCs), that the IPS cannot search. To search a CC, the second stage, called the Out-of-Plane Search (OPS), is invoked. In OPS, the boresights are tipped and are no longer normal to the sunline. Several tippings and scannings may be alternated [3]. Moreover, IPS was shown [3] to guarantee FFSL in less than 1.5 revolutions about the sunline in the case where the two spacecraft are moving in one plane that is normal to the sunline.

In this paper, we present a three-dimensional analysis of the IPS stage of the FI algorithm given in [3] and we sharpen the corresponding bound on the time needed to establish FFSL. (The upper bound is now 1.111 revolutions for $\gamma=70^{\circ}$ ). We also provide estimates of the time needed to establish FFSL and analyze the IPS stage when the two spacecraft are not constrained to move in the same plane. Essentially, the trajectory of one or more "projections" of one spacecraft on the scanning plane of another can be used to determine whether FFSL is established. The analysis of establishing FFSL during IPS is therefore reduced to analysis in a plane, and we state an equivalent, as well as a sufficient, two-dimensional condition for FFSL.

The paper is organized as follows. In Section II, we formulate the problem and present basic concepts of FI. In Section III, we explain our new approach to bounding and estimating the time needed by the basic FI algorithms to establish FFSL when the relative trajectory is in the scanning plane of the spacecraft. In Section IV, we show that testing for FFSL, for spacecraft not constrained to move in a plane, can be reduced to testing projections in one scanning plane. In Section V, we apply the methodology to the case where one spacecraft is moving parallel to the scanning plane of another, but is not contained in it. We then conclude in Section VI. 


\section{Plane Perpendicular to the sun line. Sun in front of the plane}

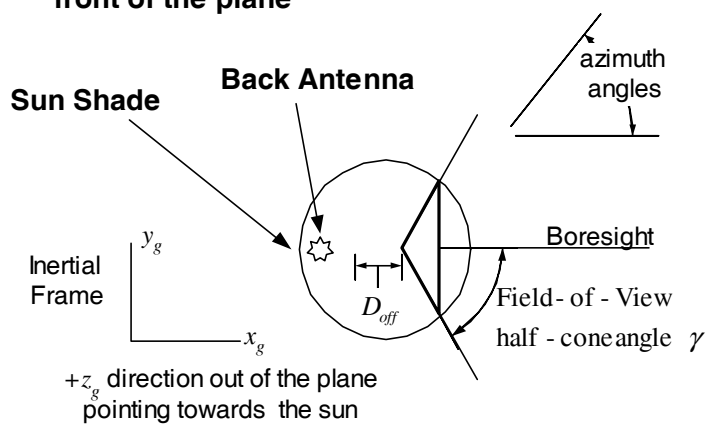

Fig. 1. Spacecraft whose sun shade lies in a plane perpendicular to the sunline.

\section{OVERVIEW OF FI ALGORITHMS}

In this paper, front-to-front sensor lock means that each of the spacecraft is within the field of View (FOV) of the AFF sensor of the other spacecraft. A plane perpendicular to the sunline is called a scanning plane. The scanning plane of a given spacecraft passes through the center of its AFF sensor. See Fig. 1. The offset $D_{\text {off }}$ is the distance between the center of mass of spacecraft and the AFF sensor mount point. This distance is subsequently neglected.

The basic FI algorithm consists, basically, of two stages: First, an In-Plane Search (IPS) stage is conducted in which the boresights of the spacecraft are rotated in the corresponding scanning planes around the sunline and at the same rate. The next stage, an Out-of-Plane Search (OPS) seeks to establish FFSL when the spacecraft are in each other's complementary cones.

We present a simplified and defeatured version of the algorithm, as described in [2,3]: First, the two spacecraft are rotated so that their sunshades are orthogonal to the sunline and their boresights are antiparallel. Then both spacecraft begin scanning (rotating about the sunline or $+z_{g}$ axis,) at the same angular rate $\Omega$ (typically $0.25 \% \mathrm{sec}$ due to the limitations of the star trackers). Front-to-front sensor lock indicates that spacecraft are within $70^{\circ}$ of total alignment between their boresights.

An inertial $x_{g} y_{g} z_{g}$ frame is here assumed where $z_{g}$ points towards the sun. A typical spacecraft has a beam pattern that looks like a cone with a half angle $\gamma$ of about $70^{\circ}$. This is illustrated in Fig. 2, where the beam of spacecraft $A$ illuminates part of spacecraft $O^{\prime} \mathrm{s}$ scanning plane. At one instant of time, this illuminated part is bounded by a hyperbola. A spacecraft is said to be scanning when the spacecraft is rotated around the line $A P$ (parallel to the sunline). If $A$ is stationary relative to $O$, the rotating hyperbola will eventually illuminate the scanning plane of $O$ with the exception of a circular region in that plane, here called the complementary footprint $(\mathrm{CF})$. This footprint can be thought of as the intersection of $O^{\prime}$ s scanning plane with a complementary cone of $A$; a cone whose axis is parallel to the sunline passing by $A^{\prime}$ s attitude rotations center. If $A$ is stationary relative to $O$, the complementary cone is the $3 \mathrm{D}$ region not illuminated by $A^{\prime}$ s beam when it scans for a full revolution. In this paper, we define the $\mathrm{CC}$ of $A$ as being attached to $A$ and moving with it; and we denote it as $\mathrm{CC}_{A}(t)$; similarly we define the complementary footprint as $\mathrm{CF}_{A}(t)=\mathrm{CC}_{A}(t) \bigcap$ scanning plane of $O$.

The intersection of $A^{\prime}$ s beam cone with $A^{\prime}$ s scanning plane results in two half lines, or rays, referred to here as the leading ray (LR) and the trailing ray (TR). The highest ray on the beam cone (as measured from $A^{\prime}$ s plane) is called the top ray, and the lowest is called the bottom ray (BR).

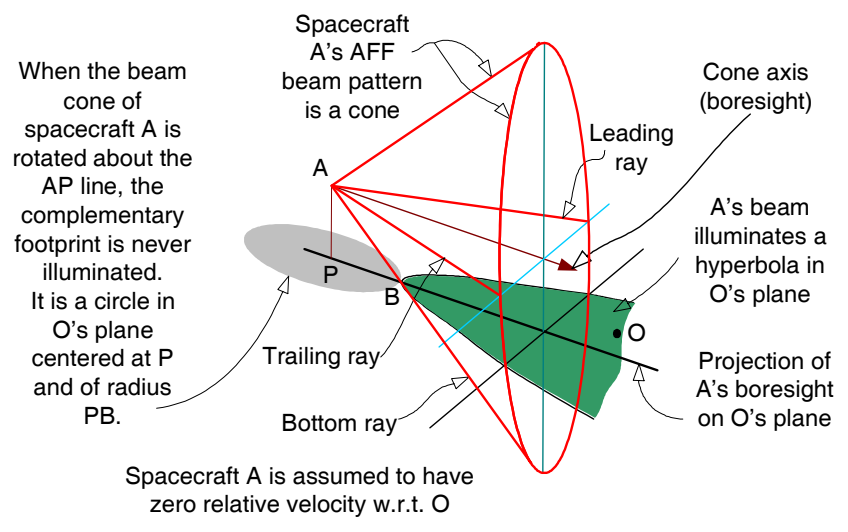

Fig. 2. Geometry of $A^{\prime}$ s AFF beam and its interaction with the scanning (principal) plane of $O$. Here $A$ and $O$ are assumed to have zero relative velocity. $A^{\prime}$ s boresight is assumed to rotate in a plane parallel to $O^{\prime} \mathrm{s}$ scanning plane.

The geometry of the scanning cone for spacecraft $A$ is illustrated in Fig. 3. The scanning rotation is about the vertical axis $z$. The boresight rotates counterclockwise in $A^{\prime}$ s scanning plane with angular speed $\Omega$. The bottom ray pierces the scanning plane of spacecraft $O$ at point $B$, here called the bottom ray projection (BRP) of $A$ on $O^{\prime}$ s plane. If spacecraft $A$ was below $O$, the top ray would pierce $O^{\prime} \mathrm{s}$ scanning plane at $B^{\prime}$, referred to as the top ray projection (TRP) of $A$ on $O^{\prime}$ s plane. The line $P B$ is the projection on $O^{\prime} \mathrm{s}$ scanning plane of $A^{\prime} \mathrm{s}$ boresight, and will rotate with angular velocity $\Omega$ about the positive $z_{g}$ axis, as well.

If after the 1.5 revolutions of IPS all spacecraft have not found one another, then the algorithm proceeds to the OPS mode, in which all angular rates are first nulled (i.e., $\Omega=$ 0 ) and subsequently a sequence of alternating tipping and scanning maneuvers are conducted to search the CCs. See [3] for details. 


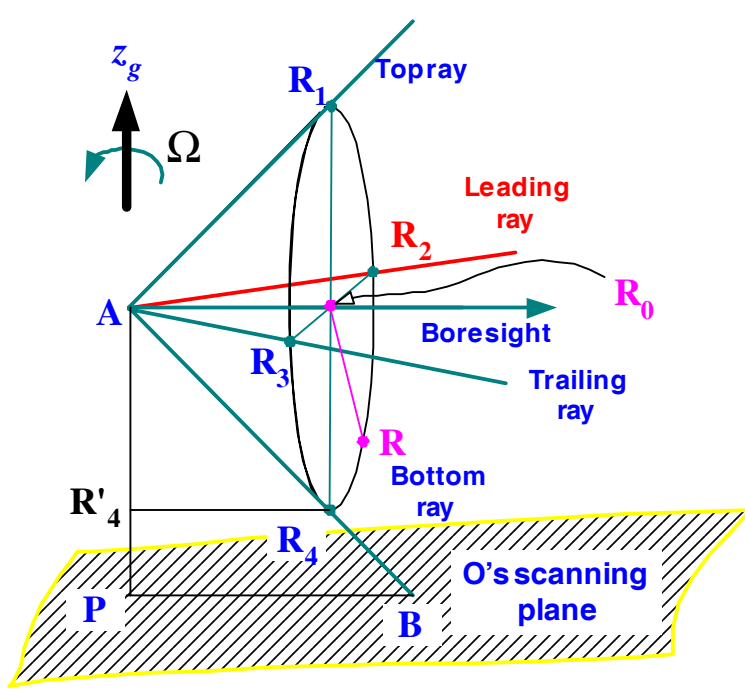

Fig. 3. The Geometry of the scanning cone of spacecraft $A$.

\section{ANALYSIS OF IPS FOR COPLANAR TRAJECTORIES}

\section{A. General approach}

The following analysis uses all or some of the following assumptions [3]:

Assumption 1: (Notation). (a) We will use "time to establish FFSL and "angle to establish FFSL", interchangeably. (b) In this paper, the global reference frame $x_{g} y_{g} z_{g}$ is different from the global frame $X Y Z$ used in [2,3]. (c) We say that $O$ illuminates $A$ if angle $\left(\overrightarrow{O A}, \vec{b}_{O}\right) \leq \gamma_{A}$, where $\vec{b}_{O}$ is the direction of the boresight of $O$. We also write $A \in \mathrm{FOV}(O)$. We say that a point $Q$ is in the Planar FOV (PFOV) of $O$ if $Q \in \operatorname{FOV}(O) \bigcap$ (scanning plane of $O$ ) and we write $Q \in \operatorname{PFOV}(O)$. (d) The boundary of a set $S$ is denoted $\partial S$ and its interior $\operatorname{int}(S)=S-\partial S$.

Assumption 2: (General Assumptions) (a) Spacecraft $A$ and $O$ do not have a priori knowledge of their relative positions. (b) No collisions occur before establishing FFSL. (c) The AFF of a spacecraft is located at the spacecraft's center of mass (There is no offset $D_{o f f}$ ). (d) Without loss of generality, assume that $O$ is stationary and that spacecraft $A$ moves with constant velocity $\vec{v}_{A}$ relative to $O$. This is accurate for deep-space applications. (e) The sunlines of all the spacecraft are parallel.

Assumption 3: All spacecraft (possibly a large number) are maneuvered such that the boresights are always either parallel or antiparallel (at any time, with or without tipping the spacecraft). During scanning, all spacecraft are rotated with a constant angular velocity of $\Omega \vec{e}_{z_{g}}$, where $\vec{e}_{z_{g}}$ is a unit vector that points to the sun (whether the spacecraft is tipped or not). Without loss of generality, the initial boresight direction of $O$ is aligned with $\vec{e}_{x_{g}}$.

Assumption 4: (Co-planar Motion Assumption) The relative position vector of $A$ with respect to $O$ is constrained to the scanning plane of $O$.
Under Assumptions 1-4, we conduct a thorough analysis of the IPS. As shown in [3], $O$ illuminates $A$ if and only if $A$ illuminates $O$. Moreover:

Theorem 1: The scanning angle $\tau$ necessary to establish FFSL is bounded by the sum of the angle $\tau_{\text {catch }}$ needed for the leading ray of $O$ to intersect (a line coinciding with) the trajectory of $A$ and the angle $\tau_{\text {scan }}$ needed by the leading ray to scan the trajectory of $A$, where $\Omega$ is the scanning angular rate.

Proof. As illustrated in Fig. 4, the leading ray of $O$ must rotate by an angle $\tau_{\text {catch }}$ (in radians) for it to intersect the trajectory of $A$, assumed to be a straight line (see Assumption 2d). Subsequently, once an intersection of the LR and the trajectory is achieved, it will take only $\tau_{\text {scan }}=$ $\pi$ radians (half a revolution) for the LR to scan/illuminate the whole straight line. Clearly the time to establish FFSL depends on $A^{\prime} \mathrm{s}$ initial position on the trajectory and its speed along it, but it must be smaller than $\pi$ radians. This is true as long as the trajectory is a straight line but is independent of the orientation of $A^{\prime} \mathrm{s}$ trajectory and its distance to $O$.
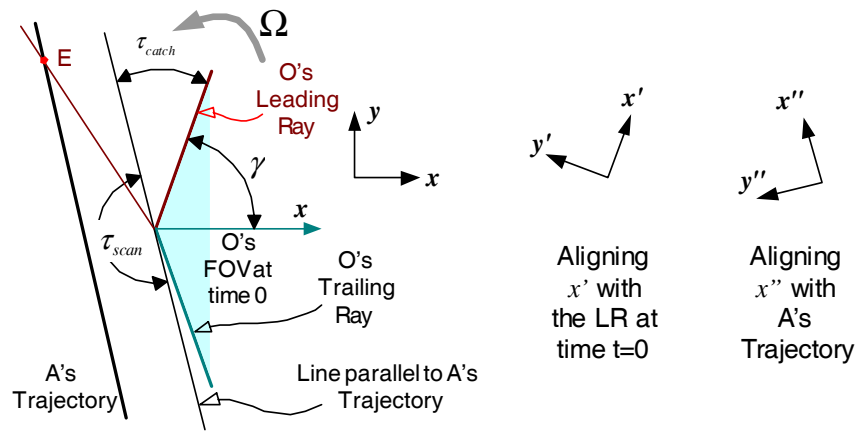

Fig. 4. Analysis of the BA algorithm in the plane. The $x^{\prime} y^{\prime}$ reference frame aligns the $x^{\prime}$ axis with the leading ray at time $t=0$ and will be used to estimate $\tau_{\text {catch }}$. The $x^{\prime \prime} y^{\prime \prime}$ reference frame aligns the $x^{\prime \prime}$ axis with the trajectory of $A$ and will be used to estimate $\tau_{\text {scan }}$.

The analysis is therefore broken down into two stages: The catching stage is analyzed using the $x^{\prime} y^{\prime}$ frame, and the trajectory scanning stage is analyzed using the $x$ " $y$ " frame. Both frames are illustrated in Fig. 4.

\section{B. Trajectory catching stage}

Here There are four cases, as illustrated in Fig. 5:

1) Trajectory intersects neither the LR nor the TR. This is true for $0 \leq \Delta \theta \leq \pi-2 \gamma$. In this case $\tau_{\text {catch }}=$ $\Delta \theta$.

2) Trajectory intersects only the TR. This is true for $\pi-2 \gamma \leq \Delta \theta \leq \pi$. In this case, points on the trajectory that are located below point $E$ in the figure are immediately visible. The remaining points on the trajectory are visible within $\tau \leq \Delta \theta+\pi$. 

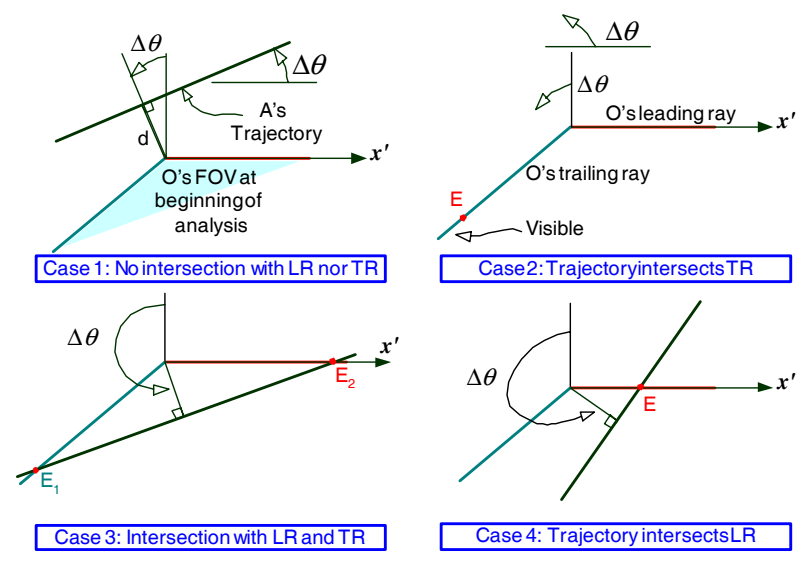

Fig. 5. The angle $\tau_{\text {catch }}$ needed for the leading ray of $O$ to catch up with the trajectory of $A$. Four cases are distinguished. All figures are in the scanning plane of $O$.

3) Trajectory intersects both the LR and the TR. This is true for $\pi \leq \Delta \theta \leq 2 \pi-2 \gamma$. One distinguishes three subcases

a) Points on the trajectory that are between $E_{1}$ and $E_{2}$ on the figure are immediately visible.

b) Points above $E_{2}$ will be scanned without catching-up delay $\left(\tau_{\text {catch }}=0\right)$ by the leading ray in a normalized time less than $\Delta \theta-\pi$, and hence $0 \leq \tau_{\text {scan }}$ (points above $\left.E_{2}\right) \leq \pi-2 \gamma$. In other words, $0 \leq \tau \leq \pi-2 \gamma$ for points above $E_{2}$.

c) For Points below $E_{1}, \tau_{\text {catch }}=\Delta \theta$, while $\tau_{\text {scan }}$ is bounded by $\pi$. Hence $\tau \leq \Delta \theta+\pi$ or simply $\tau \leq 3 \pi-2 \gamma$

4) Trajectory intersects only the LR. This is true for $2 \pi-2 \gamma \leq \Delta \theta \leq 2 \pi$. Here points below $E$ are immediately visible, while for points above $E$, $\tau_{\text {catch }}=0$ and $\tau_{\text {scan }} \leq \Delta \theta-\pi$ or simply $\tau \leq \pi$.

The worse case (longest time to establish FFSL) is Case 3c. We have thus proven the following theorem:

Theorem 2: Under Assumptions 1-4, establishing FFSL will occur before a scanning angle of $3 \pi-2 \gamma$ radians.

For a very narrow FOV, or $\gamma \approx 0$, one obtains the bound $3 \pi$ radians or 1.5 revolutions, shown in [3]. For $\gamma=70^{\circ}$, the bound is 1.111 revolutions.

\section{Trajectory scanning stage}

Now we assume that the leading ray has just caught up with $A^{\prime}$ s trajectory. In other words it is parallel to it and will intersect it in an infinitesimal time step. The geometry is illustrated in Fig. 6. The trajectory of $A$ is parallel to the leading ray of $O$, which is parallel to the $x$ axis. The distance of $O$ to $A^{\prime}$ s trajectory is $d$.

In this section we reckon time from the instant $(t=0)$ when the LR of $O$ intersects the line supporting $A^{\prime} \mathrm{s}$ trajectory. The leading ray intersects the trajectory at a

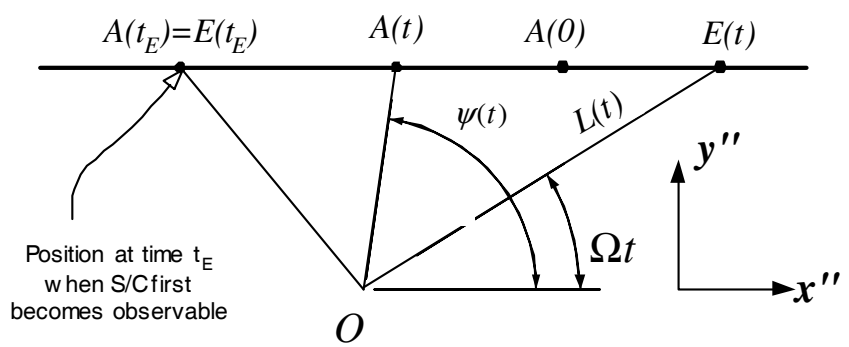

Fig. 6. Geometry of the scanning after the leading ray has just caught up with the horizontal trajectory at time $t=0$. All angles are positive counterclockwise as mesured from the $x^{\prime \prime}$ axis.

point $E(t)$ indexed on time. Clearly $x_{E}^{\prime \prime}(t) \tan \Omega t=d$ and $y_{E}(t)=d$. The position of $A$ in the reference frame is given by $y_{A}(t)=d$, and $x_{A}(t)=x_{0}+v t$ where $v$ is its horizontal speed (possibly negative). We are seeking to analyze the time needed to establish FFSL. This will occur at a time $t_{E}$ when $x_{A}\left(t_{E}\right)=x_{E}{ }^{\prime}\left(t_{E}\right)$. This implies that

$$
\left(x_{0}^{\prime \prime}+v t\right) \tan \Omega t=d
$$

where $0 \leq \Omega t \leq \pi$, and this equation can be rewritten in the nondimensional form

$$
L(\tau)=\frac{x_{0}^{\prime \prime}}{d}+\frac{v}{\Omega d} \tau=\cot \tau
$$

where $0 \leq \tau \leq \pi$. The intersections are illustrated in Fig. 7 , and they can be easily characterized.

The existence of multiple intersections can be explained as follows: The speed of $A$ along its trajectory is constant, but that of $E$ is not. Indeed, it is infinite at time $t=0$ and $t=2 \pi / \Omega$, and $E$ is slowest when it is closest to $O$. When $v<0, A$ is trying to escape from $E$. For the proper combination of initial position and speed of $A, E$ catches up with $A$ while $E$ is moving fast, then $E$ slows down and $A$ escapes, but ultimately $E$ speed up and catches up with $A$ again.

\section{TESTS FOR ESTABLISHING FFSL IN THE SCANNING PLANE}

Here Assumption 4 is lifted. Our main purpose in this section is to develop tests for establishing FFSL by considering different projections of the position of one spacecraft $A$ on $O^{\prime}$ s scanning plane.

To simplify the discussion we only consider the case where $A$ is above $O^{\prime}$ s plane. The other case is deduced by symmetry. Without loss of generality we assume that boresight of $A$ is along $\vec{b}_{A}=\vec{e}_{x}$ and by the BA requirements, $\vec{b}_{O}=-\vec{e}_{x}$. Moreover we let $\vec{e}_{z_{g}}=\vec{e}_{z}$.

Refer again to Fig. 3. The beam cone of $A$ is intersected by a vertical plane at distance $\rho \cos \gamma_{A}$ from $A$, and hence every point on the resulting intersection (circle $R_{1} R_{2} R_{4} R_{3}$ ) is equidistant to $A$. In particular, $\left\|\overrightarrow{A R_{i}}\right\|=\rho$ for $i=$ $1,2,3,4$. A typical point $R$ on the circle is shown and its 


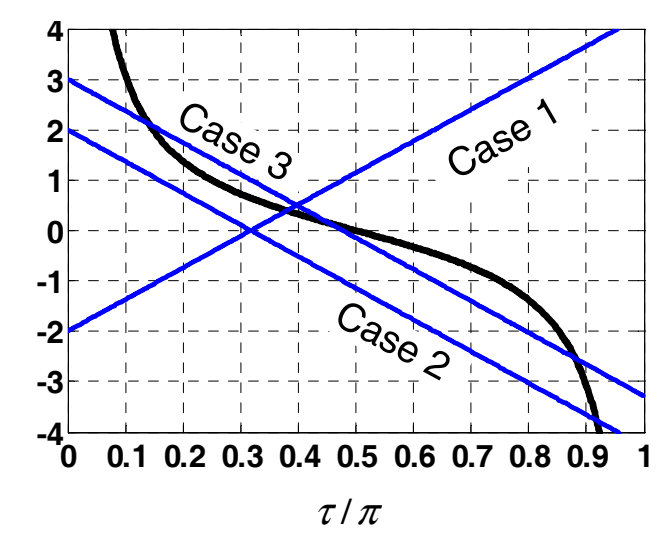

Fig. 7. Intersections between a straight line and the cotangent function.

position is characterized by the angle $\delta=\widehat{R_{2} R_{0} R}$ with $0 \leq \delta \leq 2 \pi$

We define the ratio

$$
c \triangleq \frac{z_{A}}{\rho \sin \gamma}
$$

and we note that $c \geq 0$ because $A$ is assumed to be above $O^{\prime}$ s plane. Moreover if $c>1$, then $A$ is simply too high above $O^{\prime}$ s plane to be visible by $O$. Hence we assume $0 \leq c \leq 1$ unless otherwise indicated. We note that $c=\sin \delta$ when $|c| \leq 1$. Moreover

$$
\begin{aligned}
& \overrightarrow{A R_{4}}=\vec{e}_{x} \rho \cos \gamma_{A}-\vec{e}_{z} \rho \sin \gamma_{A} \\
& \overrightarrow{R_{0} R}=\rho \sin \gamma_{A}\left(\vec{e}_{y} \cos \delta+\vec{e}_{z} \sin \delta\right),
\end{aligned}
$$

Let $R_{4}^{\prime}$ be the projection of $R_{4}$ on the line $A P$. Then using similar triangles we have $R_{4} R_{4}^{\prime} / P B=A R_{4}^{\prime} / z_{A}$ where $z_{A}=P A$ is the height of $A$ above $O^{\prime}$ s scanning plane. Then $\cos \gamma_{A} / P B=\sin \gamma_{A} / z_{A}$, or $P B=z_{A} \cot \gamma_{A}$. In short:

$$
\begin{aligned}
\overrightarrow{P A} & =\vec{e}_{z} z_{A}, \quad \overrightarrow{A B}=-\vec{e}_{z} z_{A}+\vec{e}_{x} z_{A} \cot \gamma_{A} \\
\overrightarrow{P B} & =\vec{e}_{x} z_{A} \cot \gamma_{A}
\end{aligned}
$$

In this section, we show that if the bottom ray projection (BRP) $B$ of $A$ on $O^{\prime}$ s plane belong to the planar FOV of $O$ then $O$ illuminates $A$.

Theorem 3: If $A \in \operatorname{FOV}(O)$ then $P \in \operatorname{PFOV}(O)$. Therefore if $P \notin \operatorname{PFOV}(O)$ then $A \notin \operatorname{FOV}(O)$.

Proof. Clearly $A \in \operatorname{FOV}(O)$ if angle $\left(\overrightarrow{O A}, \vec{b}_{O}\right)<\gamma$. In other words, $-\vec{e}_{x} \cdot \overrightarrow{O A} /\|\overrightarrow{O A}\|=\cos \operatorname{angle}\left(\overrightarrow{O A}, \vec{b}_{O}\right)>$ $\cos \gamma$. Or simply $-\vec{e}_{x} \cdot \overrightarrow{O A}>\|\overrightarrow{O A}\| \cos \gamma$. Now $-\vec{e}_{x} \cdot \overrightarrow{O P}$ $=-\vec{e}_{x} \cdot(\overrightarrow{O A}+\overrightarrow{A P})=-\vec{e}_{x} \cdot \overrightarrow{O A}>\|\overrightarrow{O A}\| \cos \gamma$. Hence cos angle $\left(\overrightarrow{O P}, \vec{b}_{O}\right)=\frac{-\vec{e}_{x} \cdot \overrightarrow{O P}}{\|\overrightarrow{O P}\|}>\frac{\|\overrightarrow{O A}\| \cos \gamma}{\|\overrightarrow{O P}\|}>\cos \gamma$ because $\|\overrightarrow{O A}\| \geq\|\overrightarrow{O P}\|$. Then clearly $P \in \operatorname{FOV}(O)$.
Theorem 4: If $O \in \partial \mathrm{FOV}(A)$ then $B \notin \operatorname{int}(\operatorname{PFOV}(O))$

Proof. When $O \in \partial \operatorname{FOV}(A)$ then $R \equiv O$ for some $\delta$, $\rho=\|\overrightarrow{O A}\|$, and angle $\left(\overrightarrow{A O}, \vec{b}_{A}\right)=\gamma_{A}$, and $\overrightarrow{P O}$ is given by

$$
\overrightarrow{P O}=\overrightarrow{P R}=\vec{e}_{x} \rho \cos \gamma_{A}+\vec{e}_{y} \rho \sin \gamma_{A} \cos \delta
$$

Moreover, $\overrightarrow{O B}=\overrightarrow{R B}=\overrightarrow{O P}+\overrightarrow{P B}$ and can be written

$$
\overrightarrow{O B}=\vec{e}_{x} \rho \cos \gamma_{A}(-1+c)+\vec{e}_{y}\left(-\rho \sin \gamma_{A} \cos \delta\right)
$$

where Eqs. (6) and (7) have been used. Then

$$
\begin{aligned}
\|\overrightarrow{O B}\| & =\rho \sqrt{(1-c)^{2} \cos ^{2} \gamma+\left(1-c^{2}\right) \sin ^{2} \gamma} \\
& =\rho \sqrt{1-2 c \cos ^{2} \gamma+c^{2} \cos ^{2} \gamma-c^{2} \sin ^{2} \gamma} \\
& =\rho \sqrt{(1-c)(1-c \cos 2 \gamma)}
\end{aligned}
$$

With $\vec{b}_{O}=-\vec{e}_{x}$ we can write:

$$
\begin{aligned}
\cos a_{B} & \triangleq \cos \text { angle }\left(\overrightarrow{O B}, \vec{b}_{O}\right)=-(\overrightarrow{O B} /\|\overrightarrow{O B}\|) \cdot \vec{e}_{x} \\
& =-\frac{-\rho \cos \gamma_{A}+z_{A} \cot \gamma_{A}}{\rho \sqrt{(1-c)(1-c \cos 2 \gamma)}} \\
& =\sqrt{\frac{1-c}{1-c \cos 2 \gamma} \cos \gamma \leq \cos \gamma}
\end{aligned}
$$

(assuming $0 \leq c<1$ ). Therefore $a_{B} \geq \gamma$ and hence $B \notin \operatorname{PFOV}(O)$. If $c=1$ then $a_{B}=\gamma$ and $z_{A}=\rho \sin \gamma_{A}$, and $B \in \partial \operatorname{PFOV}(O)$. Therefore $B \notin \operatorname{int}(\operatorname{PFOV}(O))$, the interior of $\operatorname{PFOV}(O)$.

The above theorem states that if $O$ and $A$ see each other, $B$ can still be outside the FOV of $O$. Indeed if $O \in$ $\partial \mathrm{FOV}(A)$, then $B$ is definitely outside the $\operatorname{PFOV}(O)$. But what if $B$ was inside $\operatorname{PFOV}(O)$ ? The following theorem shows that in this case, $A$ and $O$ see each other.

Theorem 5: If $B \in \operatorname{PFOV}(O)$, then $A \in \operatorname{FOV}(O)$.

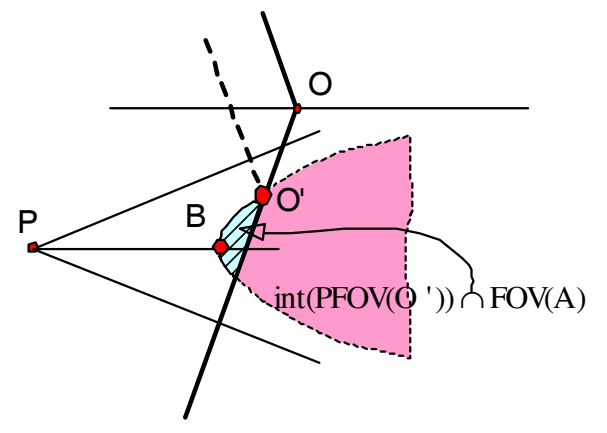

Fig. 8. The cross-hatched area is the intersection in the principal plane of $O$ of $\operatorname{PFOV}(O)$ and $\operatorname{FOV}(A)$. It defines a point $O^{\prime}$ which is a corner belonging to the boundaries of these two FOVs. The dashed line is on the boundary $\partial \mathrm{PFOV}\left(O^{\prime}\right)$.

Proof. We proceed by contradiction. Assume $B \in$ $\operatorname{int}(\operatorname{PFOV}(O))$ but $A \notin \mathrm{FOV}(O)$ and hence $O \notin \mathrm{FOV}(A)$. Then there is a point $O^{\prime} \in \partial \operatorname{FOV}(A) \cap \partial \operatorname{PFOV}(O)$ as shown in Fig. 8. Since $O^{\prime} \in \partial \mathrm{FOV}(A)$ we deduce from 
Theorem 4 that $B \notin \operatorname{int}\left(\operatorname{PFOV}\left(O^{\prime}\right)\right)$, and in particular, $B \notin \operatorname{int}\left(\operatorname{PFOV}\left(O^{\prime}\right)\right) \cap \mathrm{FOV}(A)$. By construction however, $\operatorname{int}\left(\operatorname{PFOV}\left(O^{\prime}\right)\right) \cap \operatorname{FOV}(A)=\operatorname{int}(\operatorname{PFOV}(O)) \cap \operatorname{FOV}(A)$ which is shown as the shaded area in Fig. 8. Therefore $B \notin \operatorname{int}(\operatorname{PFOV}(O)) \cap \mathrm{FOV}(A)$. But $B \in \operatorname{int}(\operatorname{PFOV}(O))$ by assumption. Then $B \notin \operatorname{FOV}(A)$ which is a contradiction, since $A B$ is the $\mathrm{BR}$ of $A$. The case where $B \in \partial \operatorname{PFOV}(O)$ can be handled by a similar argument.

Theorem 4 indicates that if $O$ and $A$ see each other, $B$ can still be outside the FOV of $O$. Let us consider such a case: $O$ and $A$ see each other, $P \in \operatorname{FOV}(O)$ but $B \notin$ $\operatorname{FOV}(O)$. The segment $P B$ must therefore intersect the $\partial \operatorname{PFOV}(O)$ at some intermediate point $M$ between $P$ and $B$; in other words,

$$
\overrightarrow{O M}=(1-\alpha) \overrightarrow{O P}+\alpha \overrightarrow{O B} \text { with } 0 \leq \alpha \leq 1
$$

and $M(\alpha)$ is indexed on $\alpha$. Moreover

$$
\overrightarrow{P M}=\overrightarrow{P O}+\overrightarrow{O M}=\alpha \overrightarrow{P B} .
$$

Theorem 6: If $P \in \operatorname{FOV}(O)$ and $B \notin \operatorname{FOV}(O)$, then $A \in \operatorname{FOV}(O)$ if and only if $M\left(\alpha^{*}\right) \in \operatorname{PFOV}(O)$ where

$$
\alpha^{*}(c)=\frac{1-\frac{1}{2} c-c^{2}+\sqrt{1-c-\frac{3}{4} c^{2}+c^{3}-\frac{1}{4} c^{4}}}{2-2.5 c^{2}}
$$

where $c=z_{A} /(\|\overrightarrow{O A}\| \sin \gamma)$.

Proof. To prove this sufficient and necessary result, one must consider the critical case $A \in \partial \mathrm{FOV}(O)$. Moving $A$ inside or outside $\operatorname{FOV}(O)$ will correspondingly move $M$ inside or outside $\operatorname{PFOV}(O)$. Therefore $R \equiv O$ and using Eqs. (7) and (8) yields

$$
\begin{aligned}
\overrightarrow{O M} / \rho= & \cos \gamma\left[-(1-\alpha)+\alpha\left(-1+z_{A} /(\rho \sin \gamma)\right)\right] \vec{e}_{x} \\
& +\sin \gamma \cos \delta((1-\alpha)-\alpha) \vec{e}_{y} \\
= & \vec{e}_{x}(-1+\alpha c) \cos \gamma+\vec{e}_{y}(1-2 \alpha) \cos \delta \sin \gamma
\end{aligned}
$$

where $\cos \delta=\sqrt{1-c^{2}}$. Then $\|\overrightarrow{O M}\|^{2}$ $=\cos ^{2} \gamma(-1+\alpha c)^{2}+\sin ^{2} \gamma\left(1-c^{2}\right)(1-2 \alpha)^{2}$. The fact that $M \in \partial \operatorname{PFOV}(0)$ implies that $-\overrightarrow{O M} \cdot \vec{e}_{x}=\|\overrightarrow{O M}\| \cos \gamma$. Squaring both sides and rearranging yields:

$$
\begin{aligned}
\cos ^{2} \gamma(1-\alpha c)^{2}= & \cos ^{2} \gamma\left(\cos ^{2} \gamma(-1+\alpha c)^{2}\right. \\
& \left.+\sin ^{2} \gamma\left(1-c^{2}\right)(1-2 \alpha)^{2}\right)
\end{aligned}
$$

which can be simplified to $0=\left(1-c^{2}\right)(1-2 \alpha)^{2}-$ $(1-\alpha c)^{2}$, a quadratic equation to be solved for the largest $\alpha$ between 0 and 1 . Expanding and rearranging then solving yields

$$
\alpha^{*}(c)=\left\{\begin{array}{l}
\frac{1-\frac{1}{2} c-c^{2}+\sqrt{1-c-\frac{3}{4} c^{2}+c^{3}-\frac{1}{4} c^{4}}}{2-2.5 c^{2}} \\
\frac{1-\frac{1}{2} c-c^{2}-\sqrt{1-c-\frac{3}{4} c^{2}+c^{3}-\frac{1}{4} c^{4}}}{2-2.5 c^{2}}
\end{array}\right.
$$

only the first of which is acceptable. Note that $\alpha^{*}(0)=$ $\alpha^{*}(1)=1$ and $\alpha^{*}(c)$ is bounded 0.8 and 1 as shown in Fig. 9.
Theorems 3-6 exhaust all the possibilities for $0 \leq c \leq$ 1 where $c=z_{A} /(\|\overrightarrow{O A}\| \sin \gamma)$. The condition $c>1$ immediately excludes the possibility of establishing FFSL.

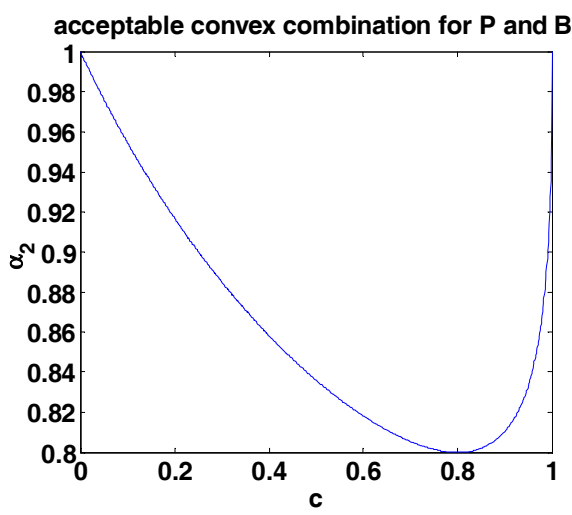

Fig. 9. Coefficient $\alpha^{*}(c)$ in Eq. (11) versus $c$.

\section{Application of tests to 3D Relative TRAJECTORIES}

To fully analyze the basic FFSL algorithm, one must consider six different cases characterizing the possible trajectories of $A$ with respect to $O$. These are: (1) $A$ is stationary and in $O^{\prime}$ s plane. (2) $A^{\prime}$ s motion is constrained in $O^{\prime}$ s scanning plane. (3) $A$ is moving in a straight line parallel to $O^{\prime} \mathrm{s}$ plane but not contained in it. (4) $A^{\prime} \mathrm{s}$ trajectory pierces the scanning plane and moves from one side of it to the other. (5) $A$ is stationary with respect to $O$ but not in its plane. (6) $A^{\prime}$ s trajectory is normal to the scanning plane.

Cases 1 and 2 have already been accounted for in Section III. Case 5, where $A$ is stationary with respect to $O$ but not in its plane, can be further broken into two subcases. In the first $A$ is not in the complementary cone of $O$, indicating that $c<1$, and hence it will be seen by $O$ during IPS. In the second subcase $A$ is in the complementary cone of $O$ and cannot be found during IPS. However, this subcase was the motivation for the OPS phase of the FFSL algorithms and $A$ will be seen by $O$ during OPS.

The other cases are considerably harder to analyze, and a complete analysis is beyond the scope of this paper. Here we will only simply consider case 3 where $A$ is moving in a straight line parallel to $O^{\prime}$ s plane but not contained in it. Then $P^{\prime}$ s trajectory is parallel to $A^{\prime}$ s. The complementary footprint, which is a circle centered at $P$ and of radius $P B$, moves within a band in $O^{\prime}$ s plane as shown in Fig. 10.

The union of all the complementary footprints of $A$ def ines the so-called Complementary Envelope (CE). In other words,

$$
\mathrm{CE}_{A}=\cup_{t} \mathrm{CF}_{A}(t)
$$

According to Theorem 5, analysis based on this band will only lead to sufficient conditions for establishing FFSL 


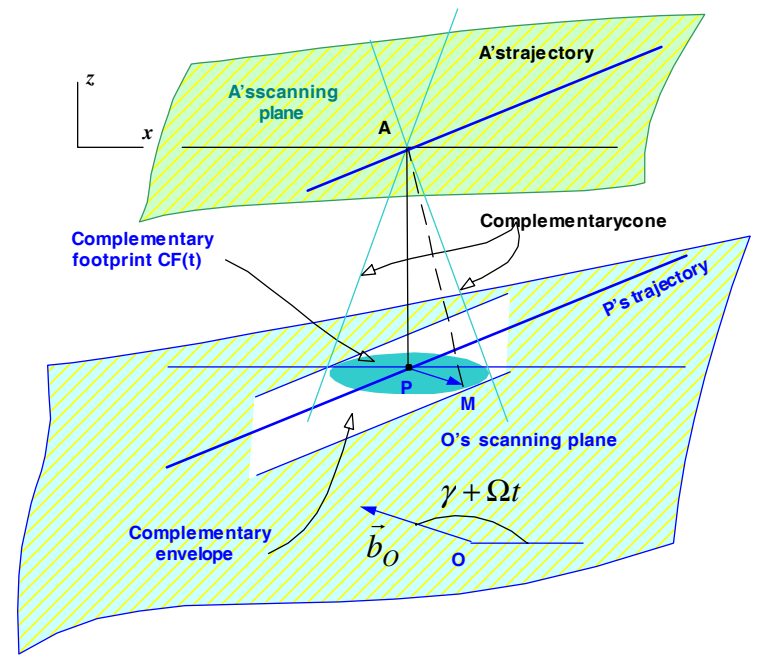

Fig. 10. Geometry of Case 3.

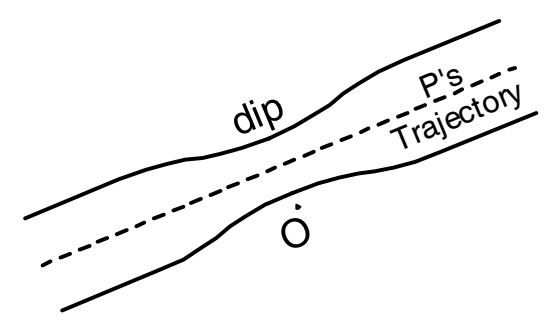

Fig. 11. When the radius of the complementary footprint is based on $P M$, a dip is introduced in the complementary envelope of $A$ near $O$. The dip cannot exceed $20 \%$ of the band width.

because the band was defined with point $B$. To obtain a necessary and sufficient condition, one must use the point $M\left(\alpha^{*}(c)\right)$ where $c$ depends on $\|\overrightarrow{O A}\|$. Therefore a slightly modified band shown in Fig. 11 must be used.

If $O \in \mathrm{CF}(t), A$ is not visible at time $t$. We call a time interval where this is true a blackout interval.

If $O \notin \mathrm{CE}$, there will be no blackout intervals. Then establishing FFSL will be guaranteed by IPS and the time to establish FFSL is the same as in the case of a single trajectory line, and it is bounded by 1.5 revolutions.

If $O \in \mathrm{CE}$, as shown in Fig. 12, there will be one "blackout" interval. The angle $\eta$ depends solely on the geometry of the relative trajectory. If the effect of the dip is ignored, the distance traveled by $P$ during the blackout is $2\|\overrightarrow{P B}\| \sin \eta$ where $\|\overrightarrow{P B}\|$ is the radius of the $\mathrm{CF}$ and is given by $P B=z_{A} \cot \gamma_{A}$. Assuming that $A$ is moving with speed $v$, the duration of the blackout is $t_{2}-t_{1} \approx$ $\frac{2}{v}\|\overrightarrow{P B}\| \sin \eta$.

If IPS is completed before the blackout or is started after the blackout, IPS will cause FFSL. If IPS is conducted completely during the blackout interval $\left[t_{1}, t_{2}\right]$, no FFSL will be established. In addition there will be many cases where only part of IPS occurs during the blackout. The arguments

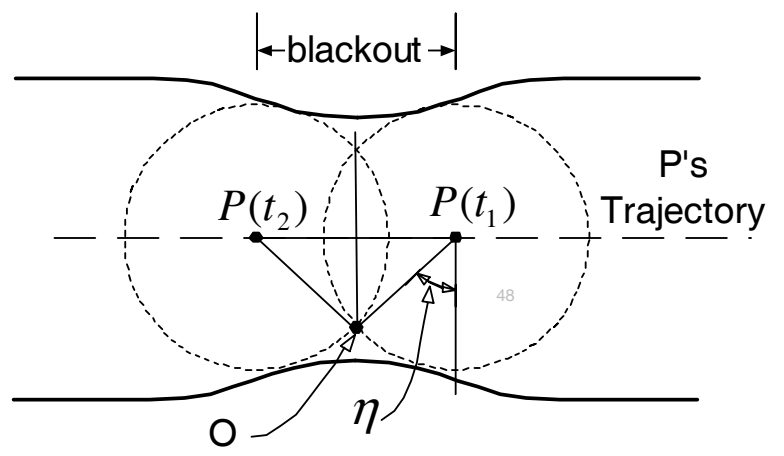

Fig. 12. When $O$ is in the CE band, there will be in general a "blackout" period.

in Sections III-B and III-C must be repeated with the caveat that if the blackout intervenes during IPS, establishing FFSL may fail. Here we simply note the possibility of failure of IPS and postpone a complete analysis. Unfortunately, OPS as described in $[2,3]$ is not guaranteed to establish FFSL: Assume that $O$ remained in the CF during all of IPS but that it moved out of it soon after IPS ended. Then the first tipping of OPS just misses, and $O$ moves out of the CF. The second tipping will not expose $A$. The secondary IPS will not expose it either. The last tip has a $2 \gamma / 2 \pi$ probability of catching it. This shows that a more extensive OPS must be developed before a complete analysis can be done.

\section{CONCLUSions}

In this paper, we extended the results in [2,3] in several directions. We provided a full three-dimensional proof and sharper bounds and estimates on the time to establish FFSL. We also showed how to analyze FFSL when the two spacecraft are not constrained to move in the same plane. The analysis is based on the concepts of bottom ray projection and the complementary envelope. We derived both a sufficient and an equivalent condition for establishing FFSL between 2 spacecraft in non-coplanar positions based on a $2 \mathrm{D}$ test. Moreover, we have highlighted a case in which the OPS proposed in [3] cannot guarantee FFSL, and therefore, will require modification.

\section{REFERENCES}

[1] S.-C. Wu and D. Kuang, "Positioning with Autonomous Formation Flyer (AFF) on Space-Technology 3," in ION GPS, Nashville, TN, 1999.

[2] F. Y. Hadaegh, D. P. Scharf, and S. R. Ploen, "Initialization of Distributed Spacecraft For Precision Formation Flying, Proceedings of the 2003 IEEE Conference on Control Applications, 23-25 June 2003, Istanbul, Turkey, pp. 1463 - 1468.

[3] D. Scharf, S. Ploen, F. Hadaegh, J. Keim and L. Phan, "Guaranteed Initialization of Distributed Spacecraft Formations," AIAA Paper 2003-5590, 2003 AIAA Guidance, Navigation, and Control Conference and Exhibit, Austin, Texas. 